CERN-TH-2017-266

January 22, 2018

\title{
The Top Mass in Hadronic Collisions
}

\author{
P. NASON \\ Theoretical Physics Department, CERN, Geneva, Switzerland, and \\ INFN, Sezione di Milano Bicocca, Milano, Italy.
}

\begin{abstract}
I discuss theoretical issues related to the top mass measurements in hadronic collisions.
\end{abstract}

Contribution to the volume

From My Vast Repertoire - The Legacy of Guido Altarelli. 


\section{Introduction}

There is something strange about the current status of top mass measurements at hadron colliders. Regarding the most precise measurements, performed by kinematic reconstruction of the full $t \bar{t}$ event, and commonly referred to as "Standard Measurements", often it is not stated clearly what is measured, in contrast with the very small error that is proudly quoted by the experimental collaborations. In some circumstances, it is claimed that what is measured is a "Monte Carlo" mass, i.e. just a parameter in the generator used to perform the analysis. But yet extensive variations of Monte Carlo parameters are performed in order to estimate errors, in a clear effort to reach something more fundamental. Other measurements, for example from the total cross section, or from some kinematic distributions, are presented collectively as "Pole Mass Measurement", that is (in the context of perturbation theory) a theoretically well defined parameter. No attempt is made to combine these pole mass measurements with the direct measurements. ${ }^{1}$

I remember having a discussion on this issue with Guido Altarelli, many years ago, at an Italian conference on LHC physics. Much time has gone by, and I do not remember the exact year and conference. A recurring argument was circulating among theoretical physicists, stating that top mass measurements at hadron colliders were just extracting the Monte Carlo mass, a parameter that did not have a well defined field theoretical definition. The argument went as follows: the top mass is extracted by fitting Monte Carlo templates to measured distributions; Monte Carlo generators have just leading order accuracy; different mass renormalization schemes cannot be distinguished at leading order, since renormalization enters at least at one loop; hence the Monte Carlo mass is not in a definite mass scheme. Oneline arguments like this are quite catchy, and tend to spread very quickly, since they do not require any specialized knowledge to be understood. I strongly disagreed with it. The measurements were clearly aiming at observables that were strongly correlated to the mass of the system of decay products, i.e. were aiming at the pole mass. And the error associated with the LO accuracy of the Monte Carlo were estimated by several methods, typically by varying parameters in the Monte Carlo, and by comparing its output to more precise next-to-leading order calculations. I expressed my disappointment about the diffusion of this "Monte Carlo mass" concept to Guido Altarelli. I told him that the argument was just used to scare the experimentalists with theoretical concept that they were not very familiar with, which was in fact an aspect of the issue that I was finding most irritating. Guido's answer was that, rather than scaring them, the argument should have made them think. I could not reply to that, since for an instant

\footnotetext{
${ }^{1} \mathrm{I}$ have heard a presentation where the speaker has jokingly introduced the subjects saying that the top is the only Standard Model particle with more than one mass.
} 
I thought that perhaps he was subtly implying that I myself should have done more thinking. This was quite typical of my exchanges with Guido. They were never free of some sort of friction. And that was certainly not the only occasion when I departed from a discussion with him with a slightly sore feeling. I must admit that I was never great at verbally arguing about physics issue, while Guido was clearly excellent at it. Yet, I was always eager to discuss with him. I had the perception (that I believe was shared by many others) that in order to settle an issue you had to convince him, since he was the uncontested authority in our field, and with the good reasons that we all know. And discussing with him always helped me to acquire a more detached and focused view of the problems. It is unfortunate that this discussion stopped at that point, since I didn't worry about this issue any more until recent time.

In this work I express my personal views on this subject, that at the moment is undeniably very controversial.

In the first section I will illustrate how and why the current status of the theoretical interpretation of top mass measurements has been reached. I will not try to fully reconstruct the historical development of all related arguments, and thus I will certainly omit quoting more than one important paper on this topic. I will rather focus on the arguments and papers that have had more influence in determining the current status. The message that I would like to convey is that, rather than finding or denying some relation of the Monte Carlo mass parameter to some well defined field theoretical parameters, we should find ways to estimate the error on the extraction of a theoretically well defined mass parameters when using a Monte Carlo generator.

In the second part of this work I will illustrate what I believe has been a small but useful progress, i.e. an improved understanding of the relation of the pole mass to the $\overline{\mathrm{MS}}$ mass. It is a self-contained subject where analytic calculations, not requiring modeling of non-perturbative and hadronization effects, can lead to a partial progress in our understanding of the top mass issues. I will expose this topic avoiding technicality as much as possible, yet discussing what I believe are its most relevant aspects.

Needless to say, I am trying to convince Guido.

\section{The top mass problem}

The top quark mass is a key parameter of the Standard Model. Its large size, of the order of the Electro-Weak scale, is associated with a Yukawa coupling of order 1, that gives important contributions, via radiative corrections, to Standard Model observables. After the Higgs boson discovery and the accurate measurement of its mass, the allowed values of the $W$-boson and top-quark masses have become strongly correlated, so that an accurate de- 
termination of both would lead to a SM test of considerable precision [1, 2]. At present there is some tension, at the $1.6 \sigma$ level, between the indirect topmass determination from electroweak precision data $(177 \pm 2.1 \mathrm{GeV})$ and the combination of direct measurements at the Tevatron and at the LHC [3], that yields $173.34 \pm 0.76 \mathrm{GeV}$. More recent determinations [4, 5, 6, 7] favour an even lower value, close to $172.5 \mathrm{GeV}$.

The top mass value is also critical in the issue of vacuum stability in the Standard Model [8, 9, 10]. At high scales, the Higgs quartic coupling $\lambda$ evolves to increasingly small values as $m_{t}$ grows, and above $m_{t}=171 \mathrm{GeV}$, i.e. very close to the present world average, $\lambda$ becomes negative at the Planck scale, rendering the electroweak vacuum meta-stable, while for $m_{t}>$ $176 \mathrm{GeV}$ the electroweak vacuum becomes unstable. The only conclusion that can be drawn from this result is that the current value of the Top and Higgs masses are such that no indication of new physics at any scale can be inferred by imposing the stability or metastability of the vacuum. On the other hand, the fact that the Higgs quartic coupling nearly vanishes at the Planck scale may have some deep meaning that we are now unable to unveil.

The abundant production of top pairs at the Large Hadron Collider (LHC) provides an opportunity for accurate top mass measurements, that are generically performed by fitting $m_{t}$-dependent kinematic distributions to Monte Carlo predictions. The most precise ones rely upon the full or partial reconstruction of the system of the $t$ and $\bar{t}$ decay products. The CMS measurement of Ref. [5], yielding the value $m_{t}=172.44 \pm 0.13$ (stat) \pm 0.47 (syst) $\mathrm{GeV}$, falls into this broad category ${ }^{2}$

In contrast with the increasing experimental precision with which the Top mass is measured at the LHC, the theoretical interpretation of the measurements seems to be in a questionable state. The so called "direct measurements", i.e. those that rely upon the reconstruction of the kinematics of the $t \bar{t}$ system, have been heavily criticized by some theorists as not possessing a clear relation between the extracted mass and a well defined quantum field theory parameter of the underlying theory.

As a consequence of that, in many experimental papers and talks, it is preferred not to qualify the measured mass parameter with a precise fieldtheoretical attribute, such as the "pole mass" or the "MS mass", and, at times, even to qualify it as a "Monte Carlo" mass [1, 3, 12]. This has led to the paradoxical situation that the most precise measurement available is not receiving the attention that it deserves from the theoretical community, and the interpretation of the measurement is left in a "limbo", with the hope that some theoretical work may clarify it in the future. At the same time, several theorists have suggested observables that allegedly overcome

\footnotetext{
${ }^{2} \mathrm{~A}$ similar measurement performed at $13 \mathrm{TeV}$ 11] yields a value of $172.25 \pm 0.08$ (stat + $J S F) \pm 0.62$ (syst) $\mathrm{GeV}$.
} 
the problems of the direct measurements. Although all these suggestions are valuable, since they can provide alternative determinations and consistency checks, none of them seem to possibly lead to an accuracy comparable with the direct determination. Furthermore, these alternative measurements are grouped in a different category by the experiments, that do not attempt to combine them with the standard measurements.

The aspect of the top mass measurement that is mostly puzzling the theoretical community, is that the top mass cannot be defined in terms of the mass of the system of its decay products. Since top is a coloured object, no final-state hadronic system can be unambiguously associated with it. On the other hand, the top mass bears certainly some relation to the mass of the system of objects arising from its decay (i.e. leptons, neutrinos and hadronic jets). The mass distribution of this system can be computed, and the top mass enters as a parameter in this computation. This is in fact the case for many parameters that are measured in high energy physics experiment. In the case of top, however, the computation in question is performed by a parton shower generator. Thus the idea that what is measured is a "Monte Carlo" mass. This idea has been expressed by several authors, but not always in the same sense: one line of thinking has to do with the perturbative accuracy of Monte Carlo generators, while others worry about the nonperturbative effects that they model. As far as the "perturbative accuracy" is concerned, the argument is essentially as follows: since shower Monte Carlo are only accurate at leading order, they cannot possibly distinguish mass parameter definitions that differ only at next-to-leading order, like the pole mass and the $\overline{\mathrm{MS}}$ mass, whose difference amounts to several GeV's.

In the following I will examine more carefully the arguments and the studies that have led to the current situation. I will show that the most disturbing aspect of the "Monte Carlo Mass" concept is its ambiguous role. In other words, it has acquired a different significance according to different authors, up to the point where it is used to support conflicting points of view.

\subsection{The "Perturbative" argument}

Fixed order theoretical calculations of (infrared safe) final state distributions can be catalogued according to their perturbative accuracy as Leading Order (LO), next-to-leading order (NLO) and so on. The top mass parameter in a theoretical calculation must be defined within a given renormalization scheme, since (divergent) perturbative corrections arise order by order in perturbation theory. For the top mass parameter, one such scheme is the pole mass scheme, that prescribes to subtract the divergent mass corrections in such a way that the pole in the quark propagator remains fixed order by order in perturbation theory. Alternatively, the $\overline{\mathrm{MS}}$ scheme prescribes to subtract the pure $1 / \epsilon$ pole in the divergent mass correction. Thus, in the $\overline{\mathrm{MS}}$ 
scheme the position of the pole in the top propagator receives corrections at all orders in perturbation theory. The two definitions lead to perturbatively equivalent theories, in the sense that there is a perturbative expression of the pole mass in terms of the $\overline{\mathrm{MS}}$ mass that allows one to translate a physical result in the pole mass scheme into the corresponding physical result in the $\overline{\mathrm{MS}}$ scheme. We notice that the $\overline{\mathrm{MS}}$ mass begins to differ from the pole mass at first order in perturbation theory, that contributes about $7.5 \mathrm{GeV}$ to the total difference.

We report below the relation between the top pole mass $m_{p}$ and $\overline{\mathrm{MS}}$ mass $m$ up to the four loops level [13],

$$
m_{p}=m+\underbrace{7.557}_{\mathrm{NLO}}+\underbrace{1.617}_{\mathrm{NNLO}}+\underbrace{0.501}_{\mathrm{N}^{3} \mathrm{LO}}+\underbrace{0.195 \pm 0.005}_{\mathrm{N}^{4} \mathrm{LO}} \mathrm{GeV} .
$$

for $m=163.643 \mathrm{GeV}$ and $\alpha_{s}^{(6)}(m)=0.1088$, in order to show the typical numbers that come into play. Thus, the "perturbative argument", if pushed to its extreme, would lead us to conclude that the relation of the extracted mass parameter to some physically well defined one has an ambiguity of the order of $10 \mathrm{GeV}$.

The earliest written record of this argument that I found is in ref. [14, where it is stated quite clearly that "the top-quark mass derived from the kinematical reconstruction does not correspond to a well defined renormalization scheme leading to a theoretical uncertainty in its interpretation. Nevertheless it is usually interpreted as the top-quark pole mass". In the same work it was proposed to extract the top mass by comparing kinematic observables in $t \bar{t}$ events involving one additional jet. Such process can be computed at NLO level, and so it must use a well defined mass renormalization scheme 3

This argument has also been used to support mass measurement based upon the $t \bar{t}$ cross section, that is now computed at NNLO order [15].

Sometimes, all the mass measurement techniques that refer to a fixed order calculation beyond the Leading Order are collected together and presented as "measurements of the top pole mass", in contrast with direct measurement where the mass scheme is never specified (see https://twiki. cern.ch/twiki/pub/CMSPublic/PhysicsResultsTOP/pole_mtop.pdf).

\subsection{The "Non Perturbative" argument}

There are claims in the literature that the difference between the top pole mass and the mass extracted in direct methods arises due to non-perturbative

\footnotetext{
${ }^{3}$ It should be recalled, however, that the difference between the pole and the $\overline{\mathrm{MS}}$ mass receives contributions beyond the NLO order that amount to more than $2 \mathrm{GeV}$. Thus, one may also argue that in an NLO calculation the pole mass parameter used there is related to a fundamental parameter of the theory with an uncertainty of at least $2 \mathrm{GeV}$.
} 
effects, that are only modelled in a shower Monte Carlo. This claim is certainly true, although the difficult bit in it is to quantify this difference. Again, this difference is used to motivate a "Monte Carlo mass" concept, that cannot be related to a well defined theoretical parameter because of these non-perturbative effects. It does not have the same meaning as the "Perturbative" difference discussed previously. But also if we restrict ourselves to this "Non Perturbative" meaning, different authors interpret it differently.

In ref. [16], the direct measurements are criticized on the basis that they reconstruct the top mass making use of jets, and jet reconstruction is affected by hadronization effects that are modelled by the shower Monte Carlo. In contrast, they propose using observables that only depend upon the lepton kinematics and that are insensitive to production dynamics, and thus should not (in their opinion) be affected by hadronization effects.

Other kind of lepton observables have also been advocated in ref. [17, There, however, an attempt is made to single out those observable that seem less affected by shower and hadronization effects, on the basis of Monte Carlo simulation study. In other words, the authors rightfully recognize that also leptonic observables may be subject to hadronization corrections, and try to quantify these effects by Monte Carlo studies.

A considerably more elaborated argument was first put forward in ref. [18], where it is stated quite explicitly that "it is not $m_{t}^{\text {pole }}$ that is being measured by the Tevatron analyses". The argument goes through several steps, that I can only summarize here. First of all, it is argued that the pole mass scheme is a poor choice, because of the presence of an ambiguity of order $\Lambda_{\mathrm{QCD}}$ associated with the mass infrared renormalon (that I will discuss later in this work), and that the so called MSr scheme avoids this problem. The MSr mass is a mass parameter that depends upon a scale $R$. It is defined using the same self-energy diagrams that contribute to the pole mass, but their contribution is limited to loop momenta of order larger than $R$. Thus, as $R$ becomes small, the MSr mass approaches the pole mass. It is argued in [18] that an MSr mass, with $R$ taken of the order of $1 \mathrm{GeV}$, should be used as mass parameter.

The argument is further developed by considering top production at high momentum in $e^{+} e^{-}$collisions at energies much larger than the top mass. It is argued that the hemisphere masses (defined by dividing the event with a plane orthogonal to the trust axis) are calculable using SCET techniques, up to the inclusion of power suppressed effects of order $\Lambda$, provided the mass parameter is the MSr mass. From this framework, implications for the standard measurements at the Tevatron are drawn. However, they suffer for the fact that in standard measurement the top is not ultrarelativistic, and the SCET factorization cannot be applied.

It must be stressed that in ref. [18] it is recognized that the Monte Carlo 
mass parameter should be quite close to the pole mass. More specifically, it is claimed that it should be close to the MSr mass evaluated at a small scale, that in turn is close to the pole mass. This point of view is very different from the "perturbative" one, that leads to differences of order $\alpha_{s} m_{t}$ between the pole and the Monte Carlo mass.

There are several followups of the work in ref. [18. In ref. [19], it was argued that, in general, an additional uncertainty of $1 \mathrm{GeV}$ should be accounted for in top mass measurements at hadron colliders. It is also argued that the top pole mass in general cannot be determined with a precision better than $1 \mathrm{GeV}$ because of the mass renormalon problem. Although it is stated somewhere in this paper that $1 \mathrm{GeV}$ "is the energy value I use in this talk for what theorists call hadronization scale", this value, quoted in the abstract and in many places in the paper, has been echoed as is in several other publications and talks, to be taken as a serious hard limit on the precision that can be achieved in the measurement of the top mass by direct methods. In subsequent works [20], Hoang and collaborators attempt to quantify a relation between the so called Monte Carlo mass and an $\mathrm{MSr}$ mass evaluated at $1 \mathrm{GeV}$. Essentially, always in the framework of highly boosted tops, they compute the jet mass using both an NNLL SCET calculation, and the Pythia8 [21] shower Monte Carlo. They absorb the difference in the two results by a shift in the Pythia8 mass parameter with respect to the MSr mass used in SCET, and argue that this shift is the difference between the Monte Carlo and the MSr mass. Indeed, they find that the Monte Carlo mass exceeds the MSr mass by about $200 \mathrm{MeV}$ with an uncertainty of about the same size. On the other hand, also the pole mass exceeds the MSr mass by a similar amount. Whatever method one adopts to resum the divergent series that gives the pole mass in terms of the MSr mass, it seems that the difference between the pole mass and the Monte Carlo mass should be significantly less than $1 \mathrm{GeV}$.

We also observe that in [20, effects that are accounted for in the SCET results (that is supposed to be NNLL accurate) and are not present in Pythia8 (that certainly cannot claim this accuracy) are absorbed into a shift in the mass. It thus seems that the mass shift they find, rather than having a universal character, should be dependent upon the chosen observable.

\subsection{Summary of the issue}

We have seen at least three arguments that are used to criticize the standard top mass measurements:

1. The perturbative argument (i.e., MC are only LO accurate)

2. A non perturbative argument: jets have non-perturbative corrections, thus we should avoid top mass observables that involve jets, (i.e., use leptonic observables). 
3. A deeper non perturbative argument: since MC's implement ad hoc models of non-perturbative effects, we should not rely upon them, since they imply an irreducible non-perturbative error. Rather, we should resort to methods that allow to treat non-perturbative corrections from first principles.

These arguments have different meaning, and in particular the first one is independent from the remaining two, since it deals with perturbative effects, while the other two address non-perturbative issues. Yet, for example, in ref. [22] it is stated that the translation from the Monte Carlo mass to a short distance mass can only be estimated within a $1 \mathrm{GeV}$ accuracy, quoting in particular [18] 4] and this statement is used to advocate that it is preferable to extract the top mass by considering observables that can be calculated in QCD at least at NLO. It is unclear why NLO precision should get rid of non-perturbative effects.

Regarding the first of the three arguments listed above, it should be reminded that one cannot simply say that a Monte Carlo has only LO accuracy. The accuracy of a Monte Carlo depends upon the observable, and there is no simple statement that can qualify it for all observables.

The production and decay dynamics of a coloured resonance in the narrow-width limit factorize in perturbation theory, and become independent, as a consequence of the fact that the resonance can propagate a long time before decaying. Thus, in the narrow width limit and in perturbation theory, there is an unambiguous definition of the system of the resonance decay products, whose mass coincides with the resonance pole mass to all orders in perturbation theory if the pole mass scheme is used. The factorization feature of perturbation theory is also implemented in Shower Monte Carlo generators, where radiation in production and decay are developed independently, and preserve the mass of the decaying resonance. Thus, up to non-perturbative effects and soft radiation of energy comparable to the resonance width (that can violate factorization because of interference between production and decay) the resonance mass appearing in the Monte Carlo can be identified with the pole mass.

One could still argue that the accuracy of the Shower Monte Carlo generator does play a role in the mass determination. Typically, in top production, the radiation generated at the production stage can enter the $b$-jet cone, or radiation from the $b$-quark can escape the jet cone, thus altering the mass of the reconstructed decay system. These effects can lead to an error on the extracted mass. This error, however, can be reduced by improving the perturbative accuracy of the Monte-Carlo, or by tuning the Monte Carlo in such a way that it better describes radiation in production and the structure of the $b$-jet. In other words, these errors are modeling errors, and their impact

\footnotetext{
${ }^{4}$ A few months later, this $1 \mathrm{GeV}$ accuracy would have gone down to $200 \mathrm{MeV}$ in ref. [20].
} 
can be estimated (and in fact is estimated by the experiments) by varying suitable parameters in the shower Monte Carlo. But none of these errors is associated to problems in the perturbative definition of the top mass.

Fixed order calculations of kinematical distributions involving top quarks are also subject to soft radiation and non-perturbative effects, and thus cannot be considered as privileged observables for pole mass determinations, to be presented in isolation from those obtained with direct measurements. If the former can be considered pole mass determinations, the same holds for the latter. Notice that this also holds for total cross section. Even if one is willing to believe that the total cross section has no linear power corrections (i.e. a correction suppressed by a single power of a hadronic scale divided by the top mass), one should remember that total cross section measurements requires extrapolations outside of a fiducial region, and fiducial regions have cuts that can introduce linear power corrections. Associated non-perturbative effects must thus be estimated also in this case.

We can also add that the accuracy of the Monte Carlo generators currently used in standard measurements make use of POWHEG or MC@NLO generators, including NLO corrections in production. Most modern Monte Carlos, like Pythia8 [21] and Herwig7 [23], include matrix element corrections to top decay, that makes them essentially (up to an irrelevant normalization factor) NLO accurate in decay. Herwig7 also implements its own POWHEG implementation of NLO corrections to top decay. Furthermore, there are recent $\mathrm{NLO}+\mathrm{PS}$ generators that implement top production and decay including finite width, non-resonant effects and interference of radiation in production and decay [24].

The second argument is based upon the general feeling that leptonic observables should not be affected by hadronization effects. It is easy, with a simple example, to convince ourselves that this may not be the case. Consider the decay of the top into a $W$ and a $b$ jet. The emission of an extra soft pion at large angle with respect to the $b$ jet can represent a typical nonperturbative effect. Such emission would always subtract an energy of the order of few hundred MeV's from the $W$, thus reducing also the lepton energy by few hundred MeV's. Thus, it is likely that linear power corrections are also present in leptonic observables, and a corresponding uncertainty should be estimated and associated with them.

The third item in the list should be regarded more carefully. It is certainly undeniable that shower Monte Carlo do not implement a solid theory of the leading power suppressed effects. It would certainly be desirable to have such a theory, or to perform top mass measurement in frameworks where such a theory is available. This seems to be the case for top mass measurements performed by a threshold scan of the $e^{+} e^{-}$cross section (see [25] and references therein), or for the recently proposed mass measurement based upon the shape of the $\gamma \gamma$ mass spectrum at the LHC [26]. While the first possibility is conditioned by the future developments of high energy 
physics experiments, the second one seems to be limited by statistics even at the high luminosity LHC phase.

We have seen that there is a sequel of papers that advocate the use of highly boosted top-quark jets, claiming that they can be computed up to the leading power suppressed correction. These approaches require validation against data. 5 full scrutiny and criticism by the theoretical community, and, most important, should be demonstrated to be practically useful, i.e. they should lead to top mass measurements with an an error smaller than the leading power suppressed effects that they claim to model correctly. At the moment, these conditions do not seem to be fulfilled.

So, the question remains about what to do with top mass measurements by direct methods at hadron colliders. It is obvious that we have an error due to non-perturbative effects that should be estimated, but it is not enough to state that it is an error of the order of $1 \mathrm{GeV}$. We should understand whether it is 1 or more, $0.5,0.2$ or less GeV's. Some authors, typically those advocating the use of boosted tops, have argued in the past for uncertainties of non-perturbative origin, of magnitude near $1 \mathrm{GeV}$, affecting the relation of the Monte Carlo mass, and also of the pole mass, to some short distance mass. As we have seen, however, the $1 \mathrm{GeV}$ figure is scarcely motivated as a hard limit. One also gets the impression that these "near a GeV" uncertainty keep decreasing with time, as more thorough analyses are performed, leaving us wondering why such a large value was ever quoted in a first place.

\subsection{What to do}

We have seen that the theoretical problems related to the determination of the top mass at hadron colliders boil down to the problem of quantifying how power suppressed corrections affect the measurement. More specifically, it has to do with the uncertainties associated with how Monte Carlos implement power suppressed effects, and how they match them to perturbative effects. These Monte Carlo uncertainties have been translated by some authors into the concept of a Monte Carlo mass.

Translating a Monte Carlo inaccuracy into a Monte Carlo mass concept has also led to the equation: the Monte Carlo implements perturbation theory at leading order, thus its mass parameter cannot be associated with a well defined mass scheme. In this second case, it is quite clear that the right question to ask should have been: what is the error on the measurement due to the fact that the Monte Carlo is only accurate at leading order. This question has in fact been asked by the experimentalists, that have studied the errors associated with the MC perturbative inaccuracies with several techniques, and by theorists, that have developed improvements in the generators to promote the accuracy of the Monte Carlo to the NLO level.

\footnotetext{
${ }^{5}$ We should remember that this is always the case in perturbative $\mathrm{QCD}$, that is a consistent framework, but is based upon some unproven assumptions.
} 
The question now is whether uncertainties due to power suppressed effects can be reliably estimated in similar ways, i.e. by varying Monte Carlo parameters, or by including plausible alternatives in the model of hadron formation that they implement. This is currently done by the experimental collaborations and by Monte Carlo developers ${ }^{6}$ Yet, with these methods the doubt remains that we may be missing something, and investigations aiming at a better understanding of power suppressed effects, and their eventual interplay with renormalons, would be welcome. At the moment, however, there is no sound argument that suggests that the Monte Carlo mass is related to some well-defined mass parameter via a systematic shift. It has been argued by some authors that, since shower Monte Carlo have a $1 \mathrm{GeV}$ cut off on soft radiation 7 their mass parameter should be identify with an MSr mass at a scale $R=1 \mathrm{GeV}$, rather than a pole mass, which yields a difference of few hundred MeV's. It should be recalled, however, that Monte Carlos implement soft radiation by ensuring that virtual effects cancel completely the real emission corrections in inclusive quantities, via a mechanism known as "shower unitarity". This mechanism implements in practice the cancellation of soft singularities. However, also finite, nonsingular soft effects are cancelled out in this way, and we should remember that self energy mass corrections are non-singular. Thus, the "Shower cut off" argument does not track down Monte Carlo effects that can convert a pole mass to an MSr one.

Notice that it is also easy to set up simple and catchy arguments to prove that the Monte Carlo mass parameter is the top pole mass. One could argue, for instance, that, since Monte Carlos implement a top propagator with a complex pole at a fixed position, that pole must be at the (complex) top pole mass, since the use of a short distance mass would yield a pole position that is blurred by the mass renormalon effects. Needless to say, also this argument is not very convincing, and it is clear that these issues require more serious thinking. However, it doesn't seem appropriate to tell the experimental community that until such thinking is done they are not allowed to tell what they are actually measuring. Rather than telling the experimentalists to wait for new theoretical developments to interpret their results, it would be far more constructive to point out to them methods to estimate the uncertainties associated with the limitations of the generator they use. For example, if the lack of radiation below the Monte Carlo cutoff scale is a concern, one should recall that this cutoff scale can be changed. One could setup a different tune of the Monte Carlo, using a different cut-off scale, and examine whether it leads to relevant differences in the extracted

\footnotetext{
${ }^{6} \mathrm{As}$ an example, the increase in the error in the $13 \mathrm{TeV}$ top mass measurement of CMS reported in 11 relative to the previous $8 \mathrm{TeV}$ measurement is due to the introduction of alternative models of colour reconnection in Pythia8 (see [27, 28]).

${ }^{7}$ We note again the loose use of the $1 \mathrm{GeV}$ figure. In Pythia8, such cutoff is by default $500 \mathrm{MeV}$.
} 
top mass. I would also like to stress that this work should not be necessarily done by the experimentalists. It can be done by theorists as well, using simplified version of top mass observables and of detector effects.

\section{The renormalon problem}

If the renormalon ambiguity on the top pole mass was as large as $1 \mathrm{GeV}$, it would make no sense to measure the top pole mass with the experimental precision that is quoted today. Fortunately, it turns out that this ambiguity is in fact much smaller. In refs. [29] and [30] the relationship of the pole mass to the $\overline{\mathrm{MS}}$ mass for the top quark is studied. They find compatible results for the central values, while for the renormalon ambiguity they quotes values of $110 \mathrm{MeV}$ and $250 \mathrm{MeV}$ respectively, both safely below currently quoted systematic errors 8

In the rest of this article I will briefly review the mass renormalon problem. I will also try to clarify why the quoted errors are so different in the two publications.

\subsection{A simplified description of the renormalon problem}

The quark mass parameter $m$ that we introduce in our Lagrangian does not necessarily coincide with the quark pole mass, which is the position of the pole in the quark propagator. More precisely it coincides with it only at zeroth order in perturbation theory. At higher orders the mass parameter requires renormalization, but this can be carried out without ever referring to the pole position, like for example when using the $\overline{\mathrm{MS}}$ prescription.

In the following I will denote with $m$ the mass parameter renormalized in the $\overline{\mathrm{MS}}$ scheme. 9 The $\mathcal{O}\left(\alpha_{s}\right)$ correction to the position of the pole in the heavy quark propagator turns out to have a linear infrared sensitivity to the scale of the momentum flowing in the loop, i.e. to yield a contribution of the form

$$
\int_{0}^{m} \mathrm{~d} l \alpha_{s}
$$

where $l$ is the scale of the loop momentum. The upper cutoff arises since we have assumed that ultraviolet divergences related to the large $l$ region have been subtracted by renormalization. Here we assume that the renormalization scale is taken equal to $m$. Thus, the pole position is given by an

\footnotetext{
${ }^{8}$ In fact, values in this range were obtained much earlier in refs. 31, 32, mostly in a bottom physics context, but since the renormalon ambiguity does not depend upon the heavy quark mass, they also apply to top.

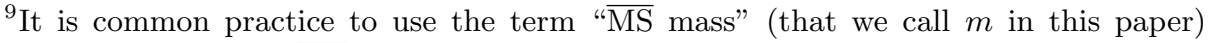
to denote the running $\overline{\mathrm{MS}}$ mass, $m(\mu)$, that depends upon a scale $\mu$, evaluated selfconsistently at the scale $\mu=m(\mu)$.
} 
expression of the form

$$
m_{\mathrm{P}}=m+N \int_{0}^{m} \mathrm{~d} l \alpha_{s}+\mathcal{O}\left(\alpha_{s}^{2}\right),
$$

where $N$ is a suitable normalization factor.

We now assume heuristically that we can obtain the higher order terms by simply writing

$$
m_{\mathrm{P}}=m+N \int_{0}^{m} \mathrm{~d} l \alpha_{s}(l)
$$

with

$$
\alpha_{s}(l)=\frac{1}{b_{0} \ln l^{2} / \Lambda^{2}}=\frac{\alpha_{s}(m)}{1-\alpha_{s}(m) b_{0} \ln m^{2} / l^{2}}=\sum_{n=0}^{\infty} \alpha_{s}^{n+1}(m) b_{0}^{n} \ln ^{n} \frac{m^{2}}{l^{2}} .
$$

Inserting (5) in (4), and recalling that

$$
\int_{0}^{m} \mathrm{~d} l \ln ^{n} \frac{m^{2}}{l^{2}}=m 2^{n} \int_{0}^{1} \mathrm{~d} x \ln ^{n} \frac{1}{x}=m 2^{n} n !
$$

we get

$$
m_{\mathrm{P}}=m+N \sum_{n=0}^{\infty} m\left(2 b_{0}\right)^{n} \alpha_{s}^{n+1}(m) n ! .
$$

The factorial growth of the coefficients of the perturbative expansion is what is called "renormalon", where the name is suggested by the fact that it arises due to the renormalization group evolution of the coupling constant. It leads to a power expansion that has zero radius of convergence. This is related to the fact that the integral in eq. (4) runs over the Landau Pole, i.e. the divergence of $\alpha_{s}(l)$ when $l=\Lambda$. The terms in the sum (7) initially decrease for $\alpha_{s}(m)$ small enough, but at some value of $n$ they start growing again. The value at which the minimum is attained is easily obtained by use of the Stirling approximation $n ! \approx \sqrt{2 \pi n} \exp (n \ln n-n)$. The term of order $n+1$ in Eq. (7) can be written as

$$
\delta m^{(n+1)}=N m \alpha_{s}(m) \sqrt{2 \pi} \exp \left[\left(n+\frac{1}{2}\right) \ln n-n+n \ln \left(2 b_{0} \alpha_{s}(m)\right)\right],
$$

that has a minimum when the derivative of the exponent with respect to $n$ vanishes

$$
\ln n+\frac{1}{2 n}+\ln \left(2 b_{0} \alpha_{s}(m)\right)=0 .
$$

Under this condition the minimal term can be written as

$$
\delta m_{\min }=N m \alpha_{s}(m) \sqrt{2 \pi} \exp \left[\frac{1}{2} \ln n_{\min }-n_{\min }-\frac{1}{2}\right] .
$$


Solving eq. (9), we get the value of $n$ at the minimum

$$
n_{\min }=\frac{1}{2 b_{0} \alpha_{s}(m)}-\frac{1}{2}+\mathcal{O}\left(\alpha_{s}(m)\right)
$$

so that

$$
\delta m_{\min } \approx N m \sqrt{\frac{\pi \alpha_{s}(m)}{b_{0}}} \exp \frac{1}{2 b_{0} \alpha_{s}}=N \sqrt{\frac{\pi \alpha_{s}(m)}{b_{0}}} \Lambda .
$$

A rough procedure to sum divergent series is to sum up the terms as long as they decrease, stopping at the minimum, that therefore gives a first indication of the uncertainty in the result.

An alternative way to deal with factorially divergent expansion is by use of the Borel transform. Given the power series

$$
f\left(\alpha_{s}\right)=\sum_{n=0}^{\infty} c_{n} \alpha_{s}^{n+1},
$$

the corresponding Borel transform is defined as

$$
B[f](t)=\sum_{n=0}^{\infty} c_{n} \frac{t^{n}}{n !}
$$

and we have formally

$$
f\left(\alpha_{s}\right)=\int_{0}^{\infty} \mathrm{d} t e^{-t / \alpha_{s}} B[f](t) .
$$

In the case of eq. (7), where $c_{n}=N\left(2 b_{0}\right)^{n} n$ !, we get

$$
m_{\mathrm{P}}-m=N m \int_{0}^{\infty} \mathrm{d} t e^{-\frac{t}{\alpha_{s}(m)}} \sum_{n=0}^{\infty}\left(2 b_{0}\right)^{n} t^{n}=N m \int_{0}^{\infty} \mathrm{d} t e^{-\frac{t}{\alpha_{s}(m)}} \frac{1}{1-2 b_{0} t} .
$$

The presence of the pole along the real axis is again a manifestation of the renormalon problem.

The Borel procedure gives an alternative, more educated method for the summation of a factorially divergent power expansion: one takes as result the principal value of the integral, and as uncertainty something proportional to the (absolute value) of the imaginary part that arises when the contour of integration is distorted above or below the singularity (at $t=1 /\left(2 b_{0}\right)$ in our case) in the complex plane. In ref. [33] it is proposed to take the absolute value of the imaginary part divided by $\pi$ as the one-sided ambiguity, that in our case leads to

$$
N m \frac{1}{2 b_{0}} e^{\frac{1}{2 b_{0} \alpha_{s}(m)}}=\frac{N}{2 b_{0}} \Lambda .
$$

In the following we will call this method for estimating the renormalon ambiguity the "Im/Pi method". In ref. [29] the Im/Pi method is adopted, 
with the motivation that it works well in context where the renormalon effect can be related to some physical observable [33], and one can check with data that it gives reliable results.

Notice that the form of the minimal term in eq. (12) differs parametrically by the one in eq. (17), due to the presence of the extra factor $\sqrt{\alpha_{s}(m)}$. In fact, in order to use the minimal term to estimate the ambiguity of the result we should also account for the fact that several terms of similar size may lie near the minimum. Starting again from eq. (7), we can expand the exponent around the minimum to get

$$
\delta m^{n+1} \sim \delta m_{\min } \exp \left[\frac{\left(n-n_{\min }\right)^{2}}{2 n_{\min }}\right] \sim \delta m_{\min }\left[1+\frac{\left(n-n_{\min }\right)^{2}}{2 n_{\min }}\right] .
$$

It is clear now that the size of the region where the terms of the series have similar size is proportional to $\sqrt{n_{\min }}$. For definiteness, let us define this region by requiring

$$
\left[1+\frac{\left(n-n_{\min }\right)^{2}}{2 n_{\min }}\right]<f, \quad \text { with } f>1 .
$$

This leads to

$$
\left|n-n_{\min }\right|<\sqrt{2(f-1) n_{\min }}
$$

and by multiplying the size of this region by the minimal term we get.

$$
\sqrt{2(f-1) n_{\min }} N \sqrt{\frac{\pi \alpha_{s}(m)}{b_{0}}} \Lambda=N \frac{\sqrt{(f-1) \pi}}{b_{0}} \Lambda
$$

This has the same parametric form of the ambiguity determined with the $\mathrm{Im} / \mathrm{Pi}$ method, and is in fact identical to it if we choose $f=1+1 /(4 \pi)$.

In the context of this oversimplified illustration of the mass renormalon problem, we also introduce the concept of the so called MSr mass [34. We define it as

$$
m_{\mathrm{MSr}}(R)=m+N \int_{R}^{m} \mathrm{~d} l \alpha_{s}(l) .
$$

or, equivalently

$$
m_{\mathrm{P}}=m_{\mathrm{MSr}}(R)+N \int_{0}^{R} \mathrm{~d} l \alpha_{s}(l) .
$$

Comparing eq. (23) to eq. (4) and (7), we see that the difference between the pole mass and the MSr mass is given by the same power expansion as the difference between the pole mass and the $\overline{\mathrm{MS}}$ mass, with the only difference that $\alpha_{s}$ is evaluated at the scale $R$ rather than the scale $m$.

The MSr mass looks like a formal interpolation between the $\overline{\mathrm{MS}}$ mass $m$ (when $R=m$ ) and the pole mass (when $R=0$ ). However, at the low end, when $\mu=l$ the integral does not exist. For $\mu>l$, on the other hand, the 
whole series is convergent, and does not manifest any factorial growth for large $n$. In fact, the coefficients grow factorially as long as $n<\log (m / \mu)$. For larger $n$, the factorial growth is damped out.

Since $m_{\mathrm{P}}$ is $\mu$ independent, we can also derive from (23) an evolution equation

$$
\mu \frac{\mathrm{d} m_{\mathrm{MSr}}(\mu)}{\mathrm{d} \mu}=-N \mu \alpha_{s}(\mu),
$$

that, unlike the typical renormalization group evolution equations, has also a linear dependence upon the scale $\mu$. This allows for the solution to have a well-defined perturbative expansion in terms of the coupling evaluated at some fixed scale, relative to its value at $\mu=0$. On the other hand, it is also associated with the factorial growth of its coefficients. Notice also that if we use the notation $m_{\mathrm{P}}(m)$ to denote the pole mass, and $m_{\mathrm{MSr}}(m, R)$ the $\mathrm{MSr}$ mass of a heavy quark with $\overline{\mathrm{MS}}$ mass $m$, we have

$$
m_{\mathrm{P}}(m)-m_{\mathrm{MSr}}(m, R)=m_{\mathrm{P}}(R)-R .
$$

In fact, the left hand side is equal to

$$
N \int_{0}^{R} \mathrm{~d} l \alpha_{s}(l)
$$

according to equation (23), and the right hand side is equal to the same quantity according to eq. (4) with $m$ replaced by $R$, and $m_{P}$ taken as the pole mass corresponding to a $\overline{\mathrm{MS}}$ mass equal to $R$.

As a last point, we can ask what happens if there are other heavy flavours (typically bottom and charm) below the top mass. The answer is quite obvious: we just replace $\alpha_{s}(l)$ in eq. (4) with a variable flavour $\alpha_{s}^{\mathrm{vf}}(l)$ that solves the evolution equation

$\mu^{2} \frac{\mathrm{d}}{\mathrm{d} \mu^{2}} \frac{1}{\alpha_{s}^{\mathrm{vf}}(\mu)}=-b_{0}^{\mathrm{vf}}(\mu), \quad b_{0}^{\mathrm{vf}}(\mu)=\frac{11 C_{a}-4 T_{f}\left[3+\theta\left(\mu-m_{c}\right)+\theta\left(\mu-m_{b}\right)\right]}{12 \pi}$.

We can immediately anticipate that the change in the mass relation will not be dramatic, since it is induced by a relatively small change in $\alpha_{s}$ in a relatively small region of the integration domain. However, the renormalon uncertainty will be determined by eq. (17) with $\Lambda$ corresponding to the 3 flavour $\Lambda$. This is easily understood, since the factorial growth at large order is controlled by increasingly small momenta, and thus cannot be sensitive to flavours with large mass.

\subsection{The full story}

The Pole Mass $m_{P}$ is given in terms of the $\overline{\mathrm{MS}}$ mass $m$ by an expansion of the form

$$
m_{P}=m\left(\mu_{m}\right)\left\{1+\sum_{n=0}^{\infty} c_{n}\left(\mu, \mu_{m}, m\left(\mu_{m}\right)\right) \alpha_{s}^{n+1}(\mu)\right\}
$$


where $m\left(\mu_{m}\right)$ is the $\overline{\mathrm{MS}}$ mass evaluated at the scale $\mu_{m}$, and $\mu$ is the renormalization scale. The coefficients have been evaluated up to the fourth order in $\alpha_{s}$ [13, 35]. For the moment we assume that we have only one massive flavour, and all other are massless. Formula (28) was originally presented in the 6-flavour scheme, where also divergences arising from the heavy flavour loops are subtracted according to the $\overline{\mathrm{MS}}$ prescription. The coupling constant is in this case the $\left(n_{l}+1\right)$-flavours (where $n_{l}$ is the number of light flavours) coupling constant. The same result can be expressed in the so called decoupling scheme, which is the scheme where divergences caused by the heavy flavour loops are subtracted at zero momentum [36], in which case the coupling constant is the $n_{l}$ flavours one.

In order to obtain the decoupling scheme formula, it is enough to express the $\left(n_{l}+1\right)$-flavours strong coupling in terms of the $n_{l}$ one, according to the standard matching formulae (see the QCD review in ref. [1]) and expand to the relevant order. Since the matching conditions are known at four loops [37, 38]), the full four loop accuracy of eq. (28) can be retained. From now on, we assume that formula (28) is expressed in the decoupling scheme. We stress that also in this case heavy fermion loops do enter in the calculation, but they are renormalized by zero momentum subtraction.

The leading IR renormalon divergence implies the following large- $n$ behaviour of the perturbative coefficients [39] and [33. ${ }^{10}$

$$
c_{n}\left(\mu, \mu_{m}, m\left(\mu_{m}\right)\right) \underset{n \rightarrow \infty}{\longrightarrow} N \frac{\mu}{m\left(\mu_{m}\right)} c_{n}^{(\text {as })},
$$

where

$$
c_{n+1}^{(\mathrm{as})}=\left(2 b_{0}\right)^{n} \frac{\Gamma(n+1+b)}{\Gamma(1+b)}\left(1+\frac{s_{1}}{n+b}+\frac{s_{2}}{(n+b)(n+b-1)}+\cdots\right)(30)
$$

The $b$ and $s_{i}$ coefficients can be found in ref. [29]. The $s_{i}$ coefficients of the sub-leading $\mathcal{O}\left(1 / n^{i}\right)$ behaviour can all be given in terms of the coefficients of the beta-function [39]. We notice that $m\left(\mu_{m}\right)$ cancels when inserting (29) in (28).

Although the proof of the form of the asymptotic expansion is nontrivial, it is not difficult to understand its properties. First of all, the leading asymptotic behaviour arises from the region of small momenta running in the loops. Since we are using a decoupling scheme, it is then natural that the heavy quark mass and the scale at which it is evaluated drop out. So, the only scale that can appear in the mass correction is $\mu$. If we neglect for the moment the mass of the light flavours, the form in eq. $(29)$ is the only one allowed by dimensional analysis. The form of eq. (30) then follows by imposing that in the derivative of the mass correction with respect to $\mu$ the factorially growing terms should cancel at high orders, consistently with the fact that factorial growth should arise from the low momentum region.

\footnotetext{
${ }^{10}$ Here we follow the notation of ref. 29 ]
} 
The coefficient $N$ cannot be computed exactly from first principles at the moment. In the large $n_{l}$ limit (both positive or negative) it assumes the form [40]

$$
\lim _{\left|n_{l}\right| \rightarrow \infty} N=\frac{C_{f}}{\pi} \times e^{\frac{5}{6}}
$$

which equals 0.97656 for $N_{c}=3\left(C_{f}=4 / 3\right)$. In ref. [41] it was noticed that the third and fourth known terms of the mass conversion formula (28) already show the renormalon asymptotic behaviour, and this can be used to infer higher order terms in the top pole mass relation. This was used in ref. [29] to extract the value of $N$ by fitting the third and fourth order coefficient of the exact calculation and give an improved determination of the mass relation including the resummation of the asymptotic terms ${ }^{11}$ The mass relation was determined by using the Borel prescription, illustrated earlier. The asymptotic expansion was evaluated by taking the principal value in the inverse Borel transform formula. Then, its first four terms where subtracted and replaced with the exact ones. The renormalon ambiguity, obtained according to the Im/Pi prescription, was determined to be $70 \mathrm{MeV}$, in the case in which the bottom and charm quark mass effects are neglected.

The procedure is modified when the charm and bottom mass effects are included. I will not describe it here in detail. I only wish to remark that in this case the renormalon ambiguity is obtained from the asymptotic expansion of the pole mass relation with three light flavours. In other words, the leading renormalon ambiguity only depends upon the number of light flavours, and nothing else. It is thus the same for the top, bottom and charm pole masses, and it was determined to be $110 \mathrm{MeV}$.

Adopting $\alpha_{s}\left(M_{Z}\right)=0.1181 \pm 0.0013$ and $m_{t}=163.508 \mathrm{GeV}$ (that yields $\alpha_{s}\left(m_{t}\right)=0.108531$ for the 5 -flavour coupling constant, the results can be summarized by the formulae

$$
\begin{aligned}
m_{c}, m_{b} \text { massless: } & \frac{m_{t, \mathrm{P}}}{m_{t}} & =1.06164_{-0.00089}^{+0.00086} \pm 0.00043 \\
m_{c}=1.3, m_{b}=4.2 \mathrm{GeV}: & \frac{m_{t, \mathrm{P}}}{m_{t}} & =1.06213_{-0.00096}^{+0.00088} \pm 0.00066,
\end{aligned}
$$

where the last error is the irreducible ambiguity in the Borel integral, obtained with the Im/Pi prescription, and the upper and lower error on the central value are the sum in quadrature of all (reducible) errors in the procedure, that are largely dominated by the uncertainty in $\alpha_{s}$.

In ref. [30], the same task was dealt with a rather different method. Before discussing it, it is useful to compare the final result with that of ref. [29]. For a top $\overline{\mathrm{MS}}$ mass of $m_{t}=163 \mathrm{GeV}$ used in [30], formulae (32) and (33) yield $m_{t, \mathrm{P}}=173.047$ and 173.127 respectively, to be compared

\footnotetext{
11 Yet again, the observation of ref. [41] was made much earlier in a bottom physics context, and fits to extract the value of $N$ were performed even before the fourth order term was known, see refs. [31, 42].
} 
with the 173.086 and 173.165 values reported on the last column of table 5 in ref. [30, in the row corresponding to the $R$ value equal to $m_{t}$. The final value they quote at the end of section 4.4 is $173.186 \mathrm{GeV}$, about $60 \mathrm{MeV}$ larger than the one obtained here using the result of ref. [29], and thus well within the uncertainty. On the other hand, they quote a larger uncertainty, of $180 \mathrm{MeV}$ when the bottom and charm masses are neglected, and of 250 $\mathrm{MeV}$ when they are taken into consideration. It is instructive to examine the source of the differences. For simplicity, I will focus upon the case of massless bottom and charm quarks.

In ref. [30], an extensive use is made of the MSr mass. In the case of massless bottom and charm, this is defined as

$$
m_{P}=m_{\mathrm{MSr}}(R)+R \sum_{n=0}^{\infty} c_{n}^{\prime}(\mu, R, R) \alpha_{s}^{n+1}(R),
$$

where $\alpha_{s}$ is defined in the 5-flavours scheme, and the coefficients $c_{n}^{\prime}$ are almost the same as those in eq. (28), since they are given by the same set of Feynmann diagrams except for those involving top loops. Was it not for this difference, we would have $m_{\mathrm{MSr}}\left(m_{\mathrm{MSr}}\right)=m(m)$. This is the first of a number of subtleties involved in the definition of the MSr mass in ref. [30]. More subtleties come into play when the effects of the bottom and charm masses are included. For the present discussion, however, these subtleties are irrelevant. The important point is that we can express $m_{\mathrm{MSr}}(R)$, for $R$ near the top mass, in terms of the $\overline{\mathrm{MS}}$ mass $m$, and, as shown in our elementary example, the MSr mass obeys an evolution equation free of renormalons, so that it can be computed at any scale $R$ below the top mass, at least for not too low values of $R$. Thus, we have the freedom to evaluate the pole mass in eq. (34) at a scale $R$ of our choice, with the first term evaluated using the evolution equation for $m_{\mathrm{MSr}}(R)$, while the second term is evaluated using some prescription to handle the factorial growth of the coefficients, that is again given by eq. (29).

The method used in [30] to estimate the asymptotic sum is as follows:

1. Define: $m_{\mathrm{P}}(n)$ to be given by eq. (34) truncated up to (and including) the $n^{\text {th }}$ order, and define $\Delta(n)=m_{\mathrm{P}}(n)-m_{\mathrm{P}}(n-1)$.

2. Find the minimal term $\Delta\left(n_{\min }\right)$ and the set $\{n\}_{f}=\{n: \Delta(n) \leq$ $\left.f \times \Delta\left(n_{\min }\right)\right\}$. The factor $f$ is defined to be "larger but close to unity", and the value $f=5 / 4$ is adopted, without further justification, in ref. [30].

3. The midpoint of the covered range of values in $m_{\mathrm{P}}(n)$ for $n \in\{n\}_{f}$ is used as central value, and the error is taken as half the size of the covered range. Scale variation is applied to the results within the range and included in the error. 

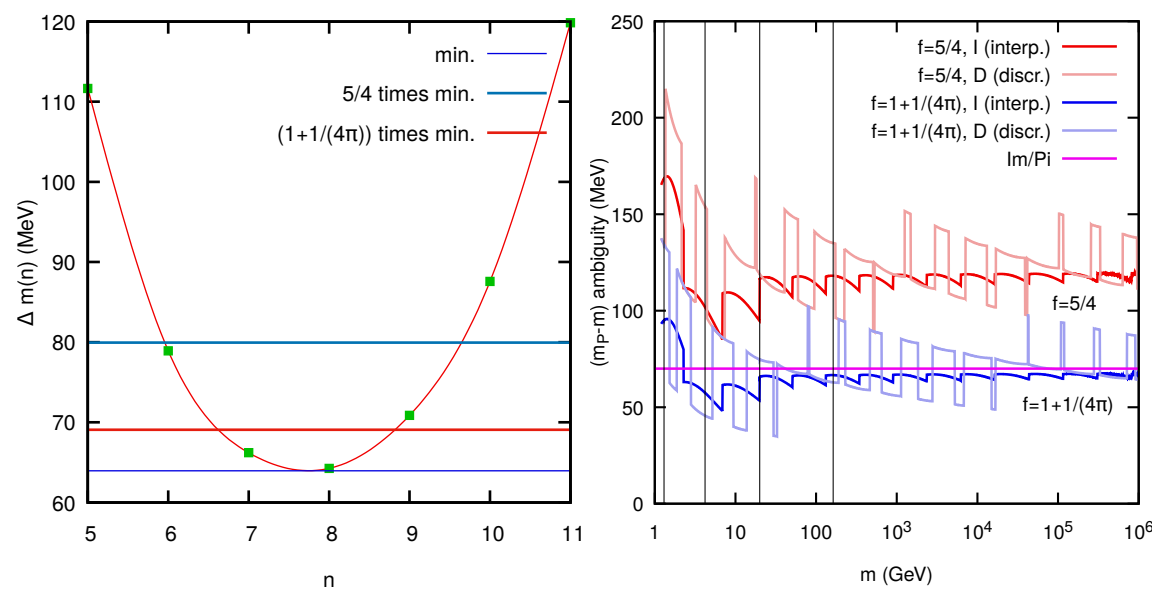

Figure 1: In the left figure, the terms in the perturbative expansion for the pole mass relation are plotted as a function of the order. In the right figure, the pole mass ambiguity as a function of the pole mass, determined using the methods D and I described in the text are plotted as a function of the $\overline{\mathrm{MS}}$ mass $m$ for two choices of the parameter $f$. The value corresponding to the $\mathrm{Im} / \mathrm{Pi}$ method is also shown. The vertical lines correspond to $m=1.3$, $4.2,20$ and $163 \mathrm{GeV}$.

First of all, we notice that this method resembles the one presented in the previous section, eq. (19), except for point 3. In fact, rather than taking half the size of the range of values covered by $m_{\mathrm{P}}(n)$, it seems more natural to take the sum of all $\Delta(n)$ divided by two ${ }^{12}$ In the following we will adopt this amendment for item 3 , and furthermore we will not consider scale variation effects, that we will discuss further on, and refer to this procedure as "method D", where D stands for "discrete". According to the discussion following eq. (17), the Im/Pi method should roughly correspond to method $\mathrm{D}$, with the choice $f=1+1 /(4 \pi)$.

The situation is illustrated in fig 1, where the size of the terms for the perturbative expansion of the pole mass relation ${ }^{13}$ are shown as a function of the order in the left plot. In the right plot, we show the ambiguity in the top pole mass as a function of the top mass $m$, taking $f=5 / 4$ or $f=1+1 /(4 \pi)$. As suggested by eq. (25), a plot analogous to the right plot in fig. 1 could be shown representing the renormalon ambiguity, for a fixed $\overline{\mathrm{MS}}$ mass, of the second term of eq. (34) as a function of $R$. Such plot is easy to obtain, and it is indistinguishable from the one shown in fig. 1. Thus I will not include it here. The reader should thus keep in mind that the plot shown in fig. 1 is

\footnotetext{
${ }^{12}$ Following literally the procedure of item 3 one would get a null error if the set $\{n\}_{f}$ consisted of a single element.

${ }^{13}$ They are taken from ref. 29, table 2, multiplied by $163 \mathrm{GeV}$ and by the factor $N=0.4616$, according to the same reference.
} 
also representative of the renormalon ambiguity as determined in ref. [30] as a function of $R$ instead of $m$, and that the vertical black lines in the figure correspond to the specific values considered there.

The pole mass ambiguity should not depend upon $m$, since it is determined by the small momentum region. However, because of the "jumpy" nature of the D prescription, we see considerable variations in the error estimate, that get worse as the mass decreases. In order to remedy to this problem, we can improve the procedure by extrapolating it to non-integer values of $n$. This approach was also used in ref. 29] in order to give an alternative estimate of the sum of the asymptotic series, and turned out to give a result that exceeded the one obtained with the Borel prescription by only $22 \mathrm{MeV}$. Here I adopt a related procedure, that has the advantage of requiring a shorter and more transparent explanation:

- Calling $m_{\mathrm{P}}(n)$ the sum of the asymptotic expansion up to (and including) the $n^{\text {th }}$ term, one finds the value of $n_{0}$ such that $m_{\mathrm{P}}\left(n_{0}\right)-$ $m_{\mathrm{P}}\left(n_{0}-1\right)$ is at a minimum. In other words, the $n^{\text {th }}$ term of the series is the smallest one.

- One finds a cubic polynomial $P(n)$ such that $P(n)=m_{\mathrm{P}}(n)$ for $n=$ $n_{0}-2, n_{0}-1, n_{0}$ and $n_{0}+1$. The polynomial $P(n)$ is taken as the extension of the series to non integer values of $n$. In particular, the value of the terms of the series at any $n$ are now given by $\mathrm{d} P(n) / \mathrm{d} n$.

- One finds $n_{\min }$ such that $\mathrm{d}^{2} P(n) / \mathrm{d} n^{2}=0$ for $n=n_{\min }$, and interprets it as the location of the minimal term. $P\left(n_{\min }\right)$ is taken as the central value for the resummed result.

- The error is taken equal to $\mathrm{d} P(n) / \mathrm{d} n$ evaluated at $n=n_{\min }$, times half the range in $n$ such that $\mathrm{d} P(n) / \mathrm{d} n$ does not exceed its minimal value by more than a factor $f$.

We call the above procedure "method I", where I stands for "interpolation". The pole mass ambiguity obtained with this method is also shown in fig. 1. where it is seen to stabilize considerably the result down to masses of a few $\mathrm{GeV}$. It can also be noticed that, in an average sense, it seems consistent with the discrete method. At very low values of the mass, however, it also becomes unreliable. This is not unexpected: at low value of the mass the minimal term occurs very early, when the series has not reached its asymptotic form. Therefore, the use of a method based upon the size of the minimal term cannot be recommended in this case. On the other hand, the $\mathrm{Im} / \mathrm{Pi}$ method always yields a constant ambiguity of $70 \mathrm{MeV}$, irrespective of the value of the mass. We notice that method I, with $f=1+1 /(4 \pi)$, is fairly consistent with the $\mathrm{Im} / \mathrm{Pi}$ method, as the simple argument following eq. (17) suggests. 
A point that needs discussion is the method adopted in ref. [30] to perform scale variation. The minimal term and the set $\{n\}_{f}$ is found for the central value of the scale, and then the scale variation is performed on the result. However, one should keep in mind that also the set $\{n\}_{f}$ can be affected by scale variation, and its change should be included, since it leads to scale compensation. This is an important point, that deserves a more detailed discussion.

If we knew the whole perturbative expansion for the pole mass relation, it would be formally independent of the renormalization scale. The series, however, is only asymptotic, and we should devise a method to sum it up. Such method better be scale independent too, so that the result of the sum is also scale independent. It is easy to show with an example, borrowed from our oversimplified model, that this is the case for the Borel method. Let us assume that the top mass relation is exactly given by the formula

$$
m_{\mathrm{P}}=m+\int_{0}^{m} \mathrm{~d} l \alpha_{s}(l)
$$

where $\alpha_{s}(l)$ is the one-loop strong coupling constant, and we assume that the only massive flavour is the top. We know how to express the above mass relation in terms of $\alpha(m)$, by using the Melling transform technique, that we now interpret as a formal, order-by-order procedure:

$$
m_{\mathrm{P}}-m=\int_{0}^{m} \mathrm{~d} l \alpha_{s}(l)=m \int_{0}^{\infty} \mathrm{d} t e^{-t / \alpha_{s}(m)} \frac{1}{1-2 b_{0} t} .
$$

We now want to express the same relation using $\alpha(\mu)$, with $\mu$ of order $m$, but different from it. This can be done as follows:

$$
\begin{aligned}
m_{\mathrm{P}}-m & =\int_{\mu}^{m} \mathrm{~d} l \alpha_{s}(l)+\int_{0}^{\mu} \mathrm{d} l \alpha_{s}(l) \\
& =\int_{\mu}^{m} \mathrm{~d} l \alpha_{s}(l)+\mu \int_{0}^{\infty} \mathrm{d} t e^{-t / \alpha_{s}(\mu)} \frac{1}{1-2 b_{0} t} .
\end{aligned}
$$

The first term can be expressed as a function of $\alpha_{s}(\mu)$ as long as $\mu>$ $\Lambda_{\mathrm{QCD}}$, and in fact it has a convergent expansion in terms of $\alpha_{s}(\mu)$, while the second term has the divergent, asymptotic expansion, still in terms of $\alpha_{s}(\mu)$. We now show that the two expressions, if the Borel integral is evaluated according to the principal value prescription, are identical, i.e. the scale variation of the series resummed according to the principal value in the Borel integral is zero. In order to prove it, we take the difference of the two formulae both regulated according to the principal value prescription. We get

$$
\int_{\mu}^{m} \mathrm{~d} l \alpha_{s}(l)+\int_{0}^{\infty} \mathrm{d} t\left[\mu e^{-t / \alpha_{s}(\mu)}-m e^{-t / \alpha_{s}(m)}\right] \frac{1}{1-2 b_{0} t}=0 .
$$



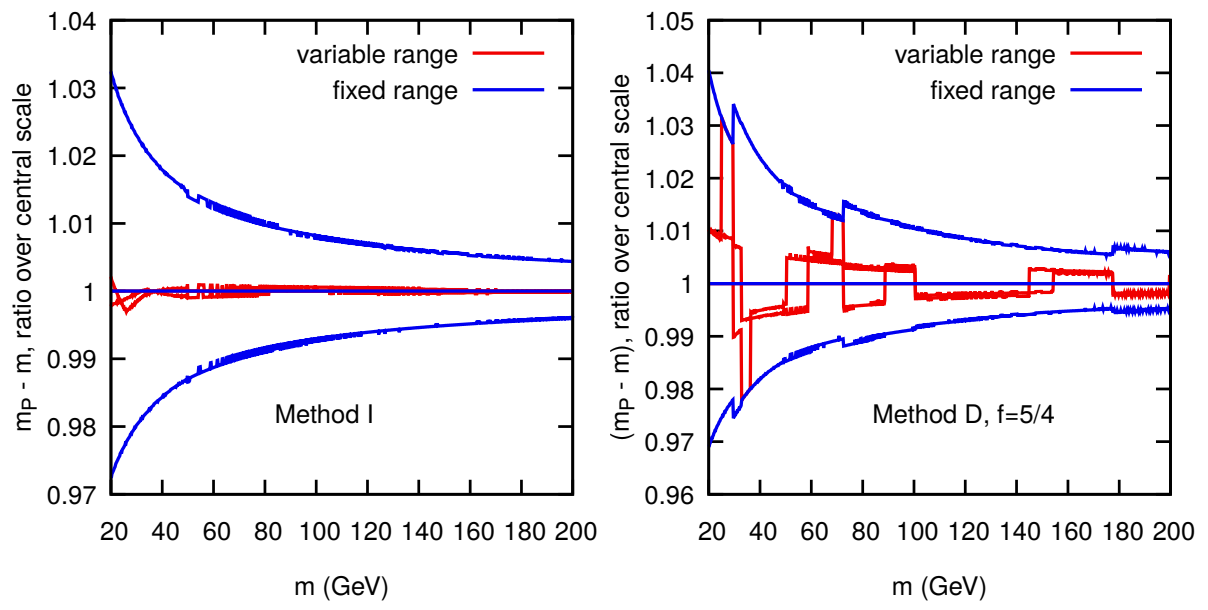

Figure 2: Scale dependence of the pole - $\overline{\mathrm{MS}}$ mass difference. The scale is varied by a factor of two above and below $m$. The curves labelled as "fixed range" are obtained by holding the position of the minimal term and of the range fixed while varying the scale; the curves labelled as "variable range" are obtained by recomputing the minimal term and the range for each scale choice. In the case of method $I$ the result does not depend upon $f$, while in the case of method $D$ a mild dependence upon $f$ is found, as can be easily understood from the definition of the method.

But now we see that the principal value prescription is no longer needed, since both terms in the square bracket become equal to $\Lambda_{\mathrm{QCD}}$ for $1-2 b_{0} t=0$. At this point we have to show that the expression vanishes. It certainly vanishes for $\mu=m$, and its derivative with respect to $\mu$, given by

$$
\begin{aligned}
& -\alpha_{s}(\mu)+\int_{0}^{\infty} \mathrm{d} t\left[e^{-t / \alpha_{s}(\mu)}\left(1+\mu \frac{\partial}{\partial \mu} \frac{-t}{\alpha(\mu)}\right)\right] \frac{1}{1-2 b_{0} t} \\
= & -\alpha_{s}(\mu)+\int_{0}^{\infty} \mathrm{d} t\left[e^{-t / \alpha_{s}(\mu)}\left(1-2 b_{0} t\right)\right] \frac{1}{1-2 b_{0} t} \\
= & -\alpha_{s}(\mu)+\int_{0}^{\infty} \mathrm{d} t e^{-t / \alpha_{s}(\mu)}=0,
\end{aligned}
$$

vanishes also, and thus we have full scale independence of the result.

In resummation methods that rely upon the minimal term, scale independence is no longer exact, since a truncation of the series is involved. But it is quite clear that the location of the minimal term must be kept variable with the scale, since it leads to scale compensation. This is illustrated in fig. 2. The figure was obtained from eq. (4), and its expansion given by the terms in eq. (7), by expressing $\alpha_{s}(m)$ as a function of $\alpha_{s}(\mu)$ (using the leading order formula) and expanding again in powers of $\alpha_{s}(\mu)$, a procedure that can be easily performed using algebraic manipulation programs. The scale $\mu$ is taken equal to $2 m$ and $m$. The series can then be resummed using 
our prescriptions D and I that rely upon the minimal term. We consider the two approaches: one where we recompute the location of the minimal term and of the range for each scale choice, and one where we do not, this last one corresponding to ref. [30]. It is clear that in both the D and I methods, further scale compensation takes place if we recompute the location of the minimal term and the range when we vary the scale. When using method I, the scale variation almost disappears, which is consistent with the observation that this method is fairly close to the $\mathrm{Im} / \mathrm{Pi}$ prescription. Again, in the D method, due to its jumpy nature, scale compensation, although considerable, is less effective. When going at very low value of the mass, both methods become quite unstable, again suggesting that it is better to avoid the use of minimal term based prescriptions in this case.

In summary, although some freedom in the determination of the renormalon ambiguity cannot be avoided, one can identify three aspects in the procedure of ref. [30] that should be amended: the choice of the parameter $f$; the insistence in evaluating the sum of the asymptotic expansion at low scales using a minimal term based, discrete method; and the procedure adopted to determine scale variation uncertainty. As far as the choice of $f$ is concerned, some justification for the chosen value is clearly missing. As far as going to low scales for determining the renormalon ambiguity, it is clear that when using resummation methods based upon the minimal term there is essentially no limit to the size of the ambiguity that one can get, since at sufficiently low scales the first term of the expansion is the minimal term, and it can become as large as one pleases because of the Landau pole. Finally, the method adopted for the scale variation uncertainty does not include effects (i.e. the displacement of the minimal point and the range) that lead to scale compensation.

As a conclusion of this discussion, we may wonder whether the issue of the size of the mass renormalon ambiguity will ever have any importance at the LHC. If we are arguing about whether the ambiguity is 110 or $250 \mathrm{MeV}$, this seems, at the moment, very unlikely. While the mass renormalon is now the only non-perturbative ambiguity that we can discuss to such detail, there are certainly other non-perturbative effects, very likely associated with renormalon uncertainty, that are much less understood, and that pose a much more serious threat to the accuracy of the top mass determination at the LHC.

\section{Conclusions}

The problem of the top mass measurement at hadron colliders is certainly a very subtle one, and has received the attention of many researchers. At the moment, there is no wide consensus in the theoretical community on how it should be dealt with. 
In the present work I have reviewed the current theoretical status, highlighting several issues that need to be resolved. In the following, I summarize my view on the subject.

In Monte Carlo generators the top decay products are distributed with a Breit Wigner distribution. Thus, the corresponding mass parameter should be qualified as the top pole mass. It is clear that Monte Carlos represent physics processes only in an approximate sense, and thus, if we use them to extract the pole mass by fitting mass sensitive distributions, the result will be subject to errors due to Monte Carlo inaccuracies. However, this is true of any calculation of a physics process. Thus, I do not accept the view that, because of these inaccuracies, one should qualify the fitted mass parameter as a "Monte Carlo mass" rather than the top pole mass. What one should instead do is to ask how the approximations and inaccuracies of the Monte Carlo propagate into mass sensitive observables, thus leading to an error on the extracted top pole mass. Observe that this is the same approach that we adopt when we perform fixed order calculations, or resummed calculations at various level of precision. There is no reason why Monte Carlo generators should be treated differently.

We can loosely separate the approximations and inaccuracies of the Monte Carlo into two categories: those that affect the perturbative part, and those that affect hadronization phenomena and their matching to the perturbative part.

In relation to the Monte Carlo inaccuracies and approximations in the perturbative part, much has been done in order to understand their impact. We have now at our disposal generators of increasing perturbative accuracy, that include only the shower, the shower with the inclusion of matrix element corrections (MEC), or the shower matched to an NLO calculation $(\mathrm{NLO}+\mathrm{PS})$ of top production and decay. By comparing them, we can assess the errors associated with the less accurate generators, and at the same time get an estimate of the remaining inaccuracies.

It is clear that for the standard measurements of the top mass, the most relevant component of the perturbative model is the formation of the $b$ jet. Thus, a shower Monte Carlo that handles production with only leading order accuracy, but performs matrix element corrections to top decay, may be quite adequate for this purpose. Thus, it is important to examine the Monte Carlo accuracy for the observable at hand. By relying upon the generic statement that Monte Carlos have only leading order accuracy, we simply miss the point.

Notice that in higher orders calculations to be used for top mass extraction in standard measurements, such as those that enter the NLO+PS generators, we must use a mass scheme such that the top mass is close enough to the pole mass (i.e. that differs from it by less than the top width), oth- 
erwise an important shift in the mass of the reconstructed top ${ }^{14}$ at the next perturbative order would systematically arise. Thus, we might as well use the pole mass scheme in these contexts.

More subtle effects, not correctly modelled with the Monte Carlo, have to do with interference of radiation in production and decay. It is unclear in this case whether the radiated parton should be considered part of the system of the top decay products (this phenomenon is a perturbative precursor of the non-perturbative color reconnection problem). The worry is that these emissions, if not implemented correctly, may induce systematic shift in the mass of the reconstructed top that would be difficult to characterize.

In recent times, techniques to deal with interference effects in production and decay within the framework of NLO+PS generators have become available [43, 44], and have been used to build a $t \bar{t}$ generator [24]. This has been compared to a generator that does not include these features [45, and it was found to yield very similar results. This can be taken as evidence that interference in production and decay has little impact on top mass measurements.

In a recent study [46] it has been shown that the new generator of ref. [24], interfaced with Pythia8, yields results on the mass of the reconstructed top that differ from the ones obtained with POWHEG-hvq interfaced with Pythia8 (that is now the default of the LHC experimental collaborations) by less than $50 \mathrm{MeV}$, if one assumes to have full access to the "particle truth" level, or less than $200 \mathrm{MeV}$, if the experimental resolution is taken into account. Results like this give us confidence that we have control over the perturbative side of our generators. They should be challenged and scrutinized by the theoretical community, also by building and trying different generators with the same or better accuracy.

Monte Carlo inaccuracies due to the modeling of non-perturbative effects, and to their interplay with renormalons, are certainly harder to examine. Even there, however, some recent progress has taken place. The calculation of the fourth order term in the relation of the pole mass to the $\overline{\mathrm{MS}}$ mass, yielding a contribution of $200 \mathrm{MeV}$, has allowed to make reliable projections on the size of higher order terms, to the point where one can be confident that the pole mass can be safely used for the LHC top mass measurements. I recognize, however, that this is just one piece of the puzzle. Other power suppressed effects may be more tricky to estimate.

Notice that estimating a "perturbative precursor" of a non-perturbative effect does not necessarily yield an upper bound on the latter. For example, the fact that at leading order interference between radiation in production and decays is small does not imply that colour reconnection effects

\footnotetext{
${ }^{14}$ Here, by "reconstructed top" I mean a system defined at the particle level that is likely to have originated from the decay of a top quark. We may define it, for example, as the hardest $b$-jet, the hardest positive lepton and the hardest neutrino of matching flavour.
} 
are small. This counter-intuitive fact may be also understood if we assume that non-perturbative effects are associated to renormalons, in which case a contribution at a given perturbative order may be followed by others that decrease less than geometrically and have all the same sign. Under these conditions, the given contribution cannot be considered a good estimate of the error due to the missing terms of higher order.

It is clear that more theoretical work is badly needed in this framework, with new ideas on how to estimate linear power suppressed effects and their interplay with renormalons. The works of ref. [18] and its followups go in the right direction, but should be extended to cover top quarks of moderate transverse momenta in order to be useful for standard top mass measurements, and should focus upon assessing the errors associated with direct measurement techniques.

There are other kinds of studies that are, in my opinion, even more urgent. Since Monte Carlos do fit the data, and since there is much arbitrarity in the way they model non-perturbative effects, studies that exploit this arbitrarity to estimate the associated uncertainties are badly needed. The inclusion of alternative models of colour reconnection in Pythia8 is one such example. These alternative models have been used by CMS, and have led to an increase of the systematic error of the top mass determination in the recent $13 \mathrm{TeV}$ measurement. Needless to say, these model should undergo further scrutiny by the theoretical community. We should examine and criticize them, and reach a consensus on whether they are acceptable, too extreme or too narrow.

I firmly believe that more studies of this sort are needed. They should proceed as follows:

1. Choose a parameter that is a matter of concern for the top mass measurement. Rather than varying a parameter, one may also consider more drastic variations, like changing a full component or the whole generator with a different one.

2. Vary the parameter and re-tune the generator (or change the generator). Restrict the range of variation of the parameter so that an acceptable tune can be achieved (or make sure that the new generator gives a good description of the data).

3. Set up a simplified framework to assess the impact on top mass measurement, and determine how much the extracted mass changes with the new setting.

Notice that this implies a lot of work, and furthermore, by proceding in this way we may end up uncovering problems that we have not yet foreseen. In other words, it takes courage to do it. However, I believe that this is the 
only way to set up a reliable method to assess the uncertainties, and gain an increased confidence on the reliability of our result.15

As an example, in the work of ref. [46] it was found that by using Herwig7 rather than Pythia8 as shower generator, the reconstructed top peak according to the particle level truth (i.e. assuming that we can detect and identify all final state particles including neutrinos and $b$ hadrons) is displaced by less than $100 \mathrm{MeV}$ when using the new generators of ref. 24] and [45, and by less than $250 \mathrm{MeV}$ when using the old POWHEG-hvq one. This result is quite remarkable, since the shower models (and, as a consequence, their interface to hadronization) and the hadronization models themselves are totally different in the two generators, and yet they cause a displacement in the top reconstructed peak that is significantly below the $1 \mathrm{GeV}$ level. The same study also finds important differences, of the order of $1 \mathrm{GeV}$, if experimental resolution effects are taken into account. This should be considered, however, a less severe problem, since in principle this error could be reduced by increasing the resolution. ${ }^{16}$

To conclude, let me say again that I am very aware that this is a controversial subject. The overall view that I have expressed should be taken as my personal one. However, several points that I made are shared by many theorist colleagues, and my current opinions have been deeply influenced by discussing with them. None of these views have been expressed in writing so far, so I have just done it here, in the hope that a more transparent debate on this subject will be started, and some wider consensus may be reached in the future.

\section{A personal note}

My last exchange with Guido was by e-mail, on the 16th of April 2015, when I learned that he had been awarded the EPS High Energy and Particle Physics Prize. I did not know of his disease at that time. I sent him a very synthetic e-mail, that had a 'CONGRATULAZIONI PER L'EPS!' in the subject, and "Era ora ... ciao, Paolo" in the body. His reply was particularly warm: "Caro Paolo, ti ringrazio moltissimo. Sei un caro amico. Saluti G". I now find this exchange particularly moving.

\section{Acknowledgements}

I wish to thank Giulia Zanderighi, Gavin Salam, Matthias Steinhauser and Michelangelo Mangano for reading the manuscript, and for providing useful suggestions.

\footnotetext{
${ }^{15}$ This can also guide us in improving the measurement method, along lines similar to those explored in ref. 47.

${ }^{16}$ Furthermore, in the same study some evidence was found that it may not be possible to fit the same data set with both generators. In other words, it may not be possible to satisfy item (2) in the above list with both generators.
} 


\section{References}

[1] Particle Data Group Collaboration, C. Patrignani et al., Review of Particle Physics, Chin. Phys. C40 (2016), no. 10100001.

[2] Gfitter Group Collaboration, M. Baak, J. Cth, J. Haller, A. Hoecker, R. Kogler, K. Mnig, M. Schott, and J. Stelzer, The global electroweak fit at NNLO and prospects for the LHC and ILC, Eur. Phys. J. C74 (2014) 3046, [1407.3792].

[3] ATLAS, CDF, CMS, Do Collaboration, First combination of Tevatron and LHC measurements of the top-quark mass, 1403.4427.

[4] ATLAS Collaboration, M. Aaboud et al., Measurement of the top quark mass in the $t \bar{t} \rightarrow$ dilepton channel from $\sqrt{s}=8$ TeV ATLAS data, Phys. Lett. B761 (2016) 350-371, 1606.02179.

[5] CMS Collaboration, V. Khachatryan et al., Measurement of the top quark mass using proton-proton data at $\sqrt{(s)}=7$ and $8 \mathrm{TeV}$, Phys. Rev. D93 (2016), no. 7 072004, 1509.04044].

[6] CMS Collaboration, Measurement of the top quark mass with lepton+jets final states in pp collisions at $\sqrt{s}=13 \mathrm{TeV}$.

[7] ATLAS Collaboration, Measurement of the top quark mass in the t $\bar{t}$ lepton+jets channel from $\sqrt{s}=8$ TeV ATLAS data, .

[8] G. Degrassi, S. Di Vita, J. Elias-Miro, J. R. Espinosa, G. F. Giudice, G. Isidori, and A. Strumia, Higgs mass and vacuum stability in the Standard Model at NNLO, JHEP 08 (2012) 098, 1205.6497.

[9] D. Buttazzo, G. Degrassi, P. P. Giardino, G. F. Giudice, F. Sala, A. Salvio, and A. Strumia, Investigating the near-criticality of the Higgs boson, JHEP 12 (2013) 089, 1307.3536.

[10] A. Andreassen, W. Frost, and M. D. Schwartz, Scale Invariant Instantons and the Complete Lifetime of the Standard Model, 1707.08124 .

[11] CMS Collaboration Collaboration, Measurement of the top quark mass with lepton+jets final states in pp collisions at $\sqrt{s}=13 \mathrm{TeV}$, Tech. Rep. CMS-PAS-TOP-17-007, CERN, Geneva, 2017.

[12] CMS Collaboration, Measurement of the ttbar production cross section in the emu channel in pp collisions at 7 and $8 \mathrm{TeV}$.

[13] P. Marquard, A. V. Smirnov, V. A. Smirnov, and M. Steinhauser, Quark Mass Relations to Four-Loop Order in Perturbative QCD, Phys. Rev. Lett. 114 (2015), no. 14 142002, 1502.01030. 
[14] S. Alioli, P. Fernandez, J. Fuster, A. Irles, S.-O. Moch, P. Uwer, and M. Vos, A new observable to measure the top-quark mass at hadron colliders, Eur. Phys. J. C73 (2013) 2438, 1303.6415.

[15] M. Czakon, P. Fiedler, and A. Mitov, Total Top-Quark Pair-Production Cross Section at Hadron Colliders Through $O\left(\frac{4}{S}\right)$, Phys. Rev. Lett. 110 (2013) 252004, 1303.6254.

[16] S. Kawabata, Y. Shimizu, Y. Sumino, and H. Yokoya, Weight function method for precise determination of top quark mass at Large Hadron Collider, Phys. Lett. B741 (2015) 232-238, 1405.2395.

[17] S. Frixione and A. Mitov, Determination of the top quark mass from leptonic observables, JHEP 09 (2014) 012, 1407.2763.

[18] A. H. Hoang and I. W. Stewart, Top Mass Measurements from Jets and the Tevatron Top-Quark Mass, Nucl. Phys. Proc. Suppl. 185 (2008) 220-226, [0808.0222].

[19] A. H. Hoang, The Top Mass: Interpretation and Theoretical Uncertainties, in Proceedings, 7th International Workshop on Top Quark Physics (TOP2014): Cannes, France, September 28-October 3, 2014, 2014. 1412.3649.

[20] M. Butenschoen, B. Dehnadi, A. H. Hoang, V. Mateu, M. Preisser, and I. W. Stewart, Top Quark Mass Calibration for Monte Carlo Event Generators, Phys. Rev. Lett. 117 (2016), no. 23 232001, 1608.01318 .

[21] T. Sjöstrand, S. Ask, J. R. Christiansen, R. Corke, N. Desai, P. Ilten, S. Mrenna, S. Prestel, C. O. Rasmussen, and P. Z. Skands, An Introduction to PYTHIA 8.2, Comput. Phys. Commun. 191 (2015) 159-177, 1410.3012.

[22] J. Kieseler, K. Lipka, and S.-O. Moch, Calibration of the Top-Quark Monte Carlo Mass, Phys. Rev. Lett. 116 (2016), no. 16 162001, 1511.00841 .

[23] J. Bellm et al., Herwig 7.0/Herwig ++ 3.0 release note, Eur. Phys. J. C76 (2016), no. 4 196, 1512.01178.

[24] T. Ježo, J. M. Lindert, P. Nason, C. Oleari, and S. Pozzorini, An $N L O+P S$ generator for $t \bar{t}$ and $W t$ production and decay including non-resonant and interference effects, Eur. Phys. J. C76 (2016), no. 12 691, 1607.04538.

[25] M. Beneke, A. Maier, T. Rauh, and P. Ruiz-Femenia, Non-resonant and electroweak $N N L O$ correction to the $e^{+} e^{-}$top anti-top threshold, 1711.10429 . 
[26] S. Kawabata and H. Yokoya, Top-quark mass from the diphoton mass spectrum, Eur. Phys. J. C77 (2017), no. 5 323, 1607.00990.

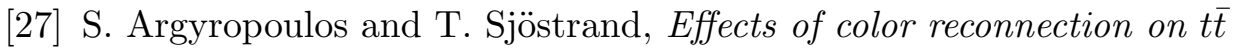
final states at the $L H C$, journal $=$. .

[28] J. R. Christiansen and P. Z. Skands, String Formation Beyond Leading Colour, JHEP 08 (2015) 003, 1505.01681.

[29] M. Beneke, P. Marquard, P. Nason, and M. Steinhauser, On the ultimate uncertainty of the top quark pole mass, Phys. Lett. B775 (2017) 63-70, [1605.03609].

[30] A. H. Hoang, C. Lepenik, and M. Preisser, On the Light Massive Flavor Dependence of the Large Order Asymptotic Behavior and the Ambiguity of the Pole Mass, JHEP 09 (2017) 099, [1706.08526].

[31] A. Pineda, Determination of the bottom quark mass from the Upsilon(1S) system, JHEP 06 (2001) 022, hep-ph/0105008.

[32] G. S. Bali, C. Bauer, A. Pineda, and C. Torrero, Perturbative expansion of the energy of static sources at large orders in four-dimensional SU(3) gauge theory, Phys. Rev. D87 (2013) 094517, 1303.3279 .

[33] M. Beneke, Renormalons, Phys. Rept. 317 (1999) 1-142, hep-ph/9807443.

[34] A. H. Hoang, A. Jain, C. Lepenik, V. Mateu, M. Preisser, I. Scimemi, and I. W. Stewart, The MSR Mass and the $\mathcal{O}\left(\Lambda_{\mathrm{QCD}}\right)$ Renormalon Sum Rule, 1704.01580.

[35] P. Marquard, A. V. Smirnov, V. A. Smirnov, M. Steinhauser, and D. Wellmann, $\overline{\mathrm{MS}}$-on-shell quark mass relation up to four loops in $Q C D$ and a general $S U(N)$ gauge group, Phys. Rev. D94 (2016), no. 7 074025, 1606.06754.

[36] J. C. Collins, F. Wilczek, and A. Zee, Low-Energy Manifestations of Heavy Particles: Application to the Neutral Current, Phys. Rev. D18 (1978) 242.

[37] Y. Schroder and M. Steinhauser, Four-loop decoupling relations for the strong coupling, JHEP 01 (2006) 051, hep-ph/0512058.

[38] K. G. Chetyrkin, J. H. Kuhn, and C. Sturm, QCD decoupling at four loops, Nucl. Phys. B744 (2006) 121-135, hep-ph/0512060.

[39] M. Beneke, More on ambiguities in the pole mass, Phys. Lett. B344 (1995) 341-347, hep-ph/9408380. 
[40] M. Beneke and V. M. Braun, Heavy quark effective theory beyond perturbation theory: Renormalons, the pole mass and the residual mass term, Nucl. Phys. B426 (1994) 301-343, hep-ph/9402364.

[41] P. Nason, Theory Summary, PoS TOP2015 (2016) 056, 1602.00443 .

[42] C. Ayala, G. Cveti, and A. Pineda, The bottom quark mass from the $\Upsilon(1 S)$ system at NNNLO, JHEP 09 (2014) 045, 1407.2128.

[43] T. Ježo and P. Nason, On the Treatment of Resonances in Next-to-Leading Order Calculations Matched to a Parton Shower, JHEP 12 (2015) 065, 1509.09071.

[44] R. Frederix, S. Frixione, A. S. Papanastasiou, S. Prestel, and P. Torrielli, Off-shell single-top production at NLO matched to parton showers, JHEP 06 (2016) 027, [1603.01178].

[45] J. M. Campbell, R. K. Ellis, P. Nason, and E. Re, Top-pair production and decay at NLO matched with parton showers, JHEP 04 (2015) 114, 1412.1828 .

[46] S. F. Ravasio, T. Jezo, P. Nason, and C. Oleari, A Theoretical Study of Top-Mass Measurements at the LHC Using NLO+PS Generators of Increasing Accuracy, 1801.03944.

[47] A. Andreassen and M. D. Schwartz, Reducing the Top Quark Mass Uncertainty with Jet Grooming, JHEP 10 (2017) 151, 1705.07135. 